\title{
BMJ Open The role of team climate in improving the quality of chronic care delivery: a longitudinal study among professionals working with chronically ill adolescents in transitional care programmes
}

\author{
Jane M Cramm, Mathilde M H Strating, Anna P Nieboer
}

To cite: Cramm JM, Strating MMH, Nieboer AP. The role of team climate in improving the quality of chronic care delivery: a longitudinal study among professionals working with chronically ill adolescents in transitional care programmes. BMJ Open 2014:4:e005369. doi:10.1136/bmjopen-2014005369

- Prepublication history for this paper is available online. To view these files please visit the journal online (http://dx.doi.org/10.1136/ bmjopen-2014-005369).

Received 1 April 2014 Revised 29 April 2014 Accepted 30 April 2014

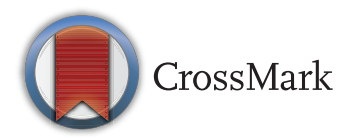

Institute of Health Policy and Management, Erasmus University Rotterdam, Rotterdam, The Netherlands

Correspondence to Dr Jane Murray Cramm; cramm@bmg.eur.nl

\section{ABSTRACT}

Objectives: This study aimed to (1) evaluate the effectiveness of implementing transition programmes in improving the quality of chronic care delivery and (2) identify the predictive role of (changes in) team climate on the quality of chronic care delivery over time. Settings: This longitudinal study was undertaken with professionals working in hospitals and rehabilitation units that participated in the transition programme 'On Your Own Feet Ahead!' in the Netherlands.

Participantss: A total of $145 / 180$ respondents $(80.6 \%)$ filled in the questionnaire at the beginning of the programme (T1), and 101/173 respondents (58.4\%) did so 1 year later at the end of the programme (T2). A total of $90(52 \%)$ respondents filled in the questionnaire at both time points. Two-tailed, paired $t$ tests were used to investigate improvements over time and multilevel analyses to investigate the predictive role of (changes in) team climate on the quality of chronic care delivery. Interventions: Transition programme.

Primary outcome measures: Quality of chronic care delivery measured with the Assessment of Chronic Illness Care Short version (ACIC-S).

Results: The overall ACIC-S score at T1 was 5.90, indicating basic or intermediate support for chronic care delivery. The mean ACIC-S score at T2 significantly improved to 6.70 , indicating advanced support for chronic care. After adjusting for the quality of chronic care delivery at T1 and significant respondents' characteristics, multilevel regression analyses showed that team climate at $\mathrm{T} 1(\mathrm{p}<0.01)$ and changes in team climate $(p<0.001)$ predicted the quality of chronic care delivery at $\mathrm{T} 2$.

Conclusions: The implementation of transition programmes requires a supportive and stimulating team climate to enhance the quality of chronic care delivery to chronically ill adolescents.

\section{BACKGROUND}

The importance of improving the quality of chronic care delivery to adolescents with chronic conditions has been increasingly

\section{Strengths and limitations of this study}

- The first strength of this study is the longitudinal design to assess the predictive role of (changes in) team climate on the quality of chronic care delivery over time.

- The second strength of the study is the evaluation of a programme to improve transitional care for adolescents with a variety of conditions, not just a single condition.

- The most important limitation of this study is that we did not investigate the predictive role of (changes in) team climate on the quality of chronic care delivery over time among professionals not participating in a quality improvement programme. Although we found that the quality of chronic care delivery improved over time after the implementation of a combination of transitional interventions and participation in a quality improvement programme, we do not know whether this improvement was larger than in teams not enrolled in such a programme or compared with the implementation of a single intervention.

- The second limitation is that other factors may additionally explain the improved quality of care delivery such as the unmet education and training needs of professionals, lack of organisational and management support and the quality of communication and coordination between professionals. Future research is necessary to unravel their predictive nature for quality of care.

- The third limitation is that our findings provide no insight into which (set(s) of) intervention(s) had the greatest impact on quality of care.

acknowledged, ${ }^{1-7}$ leading to the development of transitional care to bridge the gap between paediatric and adult care. ${ }^{8-12}$ Blum $e t a l^{7}$ defined the transition of care as the purposeful, planned movement of adolescents and young adults with chronic physical 
and medical conditions from child-centred to adultoriented healthcare systems. This transition involves a multifaceted, active process attending to the medical, psychosocial, educational and vocational needs of chronically ill adolescents. Research has shown that the delivery of effective and high-quality chronic care requires comprehensive system changes that entail more than simply implementing interventions or adding features to the existing acute-focused system. ${ }^{13-15}$ Many adolescents, however, have reported poor experiences with transitional care delivery, ${ }^{16}$ resulting in stress and anxiety. Furthermore, negative experiences with chronic care delivery are expected to result in suboptimal use of care, non-compliance to treatment and loss to follow-up. ${ }^{17} 18$

Wagner et $a l^{19}$ developed the chronic care model to guide quality improvement in chronic care delivery and the shift to care that is organised, structured, planned, patient centred and proactive through a combination of effective multidisciplinary teams and planned interactions with patients. The four core elements of the model within healthcare organisations are (1) self-management support, (2) delivery system design, (3) decision support for professionals and (4) the use of patient registries and other supportive clinical information systems. ${ }^{19} 20$ Self-management support refers to collaboratively helping patients and families to acquire the skills and confidence to self-manage a chronic condition, ${ }^{21}$ which is also considered an essential element of transitional care. ${ }^{22}$ In case of transitional care, it is important to focus on building the capacity of chronically ill adolescents to engage in a developmentally appropriate level of selfmanagement of their condition. Examples of adolescentspecific interventions are use of a self-management tool to assess transfer readiness, ${ }^{22} 23$ implementing an individual transition plan (ITP) and challenging adolescents to attend consultations independently (without parents present). ${ }^{24}$ Delivering high-quality chronic illness care demands planning and the coordinated actions of professionals not only from various disciplines, such as between nurses, medical doctors and physical therapists, but also between paediatric and adult professionals in the case of transitional care. Planned visits and regular follow-up with these multiple professionals are essential within the delivery system design to effectively monitor and manage the chronic condition. ${ }^{14}{ }^{15}$ Designing and implementing a transition (outpatient) clinic is expected to improve multidisciplinary collaboration between paediatric and adult care. ${ }^{24}$ Another example is appointing a transition coordinator in charge of transitional care for chronically ill adolescents. ${ }^{22}{ }^{24}$ Clinical protocols and guidelines (eg, a transition protocol for the transition from paediatric to adult care) for optimal chronic care delivery are needed and should be integrated into daily practice to provide decision support for professionals. $^{21}$ Clinical information systems provide support and remind professionals to comply with guidelines and protocols, such as by supplying feedback on chronic illness measures (eg, lipid or glucose level) and registries for planning individual patient care ${ }^{151921}$ in an individual treatment and/or transition plan.

Evidence clearly shows that care delivery based on the chronic care model is effective. Pearson et $a l^{25}$ for example, found evidence suggesting that the chronic care model is a useful framework for quality improvement. It leads to more positive experiences of quality of care delivery as perceived by professionals as well as chronically ill patients. ${ }^{26}$ In a meta-analysis on evidence of the chronic care model, Tsai $e t a l^{27}$ provided strong evidence of significant improvements in process outcome measures. Others found that it prevents disease complications $^{28}$ and improves patients' health behaviours. ${ }^{29}$ Furthermore, these improvements promote sustainability of programmes. ${ }^{30} \mathrm{~A}$ recent literature review reaffirmed the notion that successful improvement strategies in chronic disease care are consistent with the concept of the chronic care model. ${ }^{20}$ No research until now, however, has investigated the effectiveness of transitional care programmes in improving the quality of chronic care delivery over time, as described in Wagner's chronic care model. ${ }^{19}$ Therefore, the first aim of this study was to evaluate the effectiveness of implementing transition programmes in improving the quality of chronic care delivery, as described by the four key elements of the chronic care model.

The quality of chronic care delivery largely depends on the existence of prepared and proactive multidisciplinary teams sharing responsibility for the provision of care to their patients. ${ }^{31}$ Research, however, shows that professionals involved in transitional care identify several unmet educational and training needs as main barriers to provide age-appropriate and developmentally appropriate care to chronically ill adolescents. ${ }^{32}$ Wellfunctioning teams comprising professionals from diverse backgrounds are at the core of transition programmes, who all should have the skills and objectives needed to provide high-quality transitional care. One of the most important characteristics of such teams is their team climate. ${ }^{33}$ Research, for example, has shown that team climate is related to access to care delivery, patient satisfaction and team effectiveness. ${ }^{34} 35$ A team's performance may be facilitated or hindered by team climate. ${ }^{33} 36$ Team climate refers to professionals' shared knowledge and perceptions of the types of behaviour and action that are rewarded and supported by the team's policies, practices and procedures. ${ }^{36}$ Previous research has indeed shown that team climate was positively related to high-quality care delivery through the sharing of objectives, commitment and support among professionals. ${ }^{34} 37$ Therefore, we expected that teams whose multidisciplinary team members agree on objectives and proactively participate in decision-making, are committed to achieving the highest possible standards of chronic care delivery and receive support from other team members would be more likely to be able to improve the quality of chronic care delivery to chronically ill adolescents. The longitudinal relationship between team climate and 
quality of care delivery to chronically ill adolescents has not been studied yet. Therefore, the second aim of this study was to investigate the predictive role of team climate and changes therein on the quality of chronic care delivery over time.

\section{METHODS}

\section{Setting and design}

This longitudinal study was undertaken with professionals working in hospitals and rehabilitation units that implemented transition programmes for adolescents with chronic conditions in the Netherlands called 'On Your Own Feet Ahead!'. ${ }^{38-40}$ The 'On Your Own Feet Ahead!' programme follows the Breakthrough Series improvement and implementation strategy. ${ }^{41}$ Strong features of this strategy are the efficient use of participating experts and the exchange of best practices for improvement. Key interventions implemented for each adolescent patient within this programme were: (1) use of a transition protocol and joint mission on transitional care between paediatric and adult care, (2) appointment of a transition coordinator, (3) use of an individual transition plan, (4) multidisciplinary consultation on transition and/or implementation of a transitional (outpatient) clinic and (5) challenging the adolescent to be alone in the consultation room. The transition programme was implemented in hospitals and rehabilitation centres targeting several patient populations: adolescents with type I diabetes; juvenile rheumatoid arthritis; cystic fibrosis; kidney conditions, such as renal insufficiency/kidney failure or kidney transplantation; urological conditions, such as imperforate anus with malfunctions in the anus, rectum, urethra and bladder, or exstrophy of the bladder (a congenital anomaly in which part of the urinary bladder is present outside the body); and neuromuscular disorders (with the use of home ventilation). This study was approved by the ethics committee of the Erasmus University Medical Centre, Rotterdam, the Netherlands.

\section{Data collection and measures}

Project leaders from 29 teams selected 4 to a maximum of 10 team members (depending on the type of setting) to fill in the questionnaire used in this study. Team members were selected by project leaders based on their involvement in transitional care. In rehabilitation centres, more team members were involved since in these settings more services are involved in care for adolescents. Each selected team member received a questionnaire by mail at the beginning of the programme (T1) and 1 year later at the end of the programme (T2). A total of 145/180 respondents $(80.6 \%$ response rate) representing all 29 teams filled in the questionnaire at the beginning of the programme (T1), and 101/173 (58.4\% response rate) representing 27 teams 1 year later at the end of the programme (T2). A total of $90(52.0 \%)$ respondents filled in the questionnaire at both time points.
The Assessment of Chronic Illness Care Short version (ACIC-S) is a comprehensive tool developed in line with Wagner's chronic care model to assess the quality of chronic care delivery. ${ }^{42}$ In contrast with traditional disease-specific tools, such as the measurement of glycated haemoglobin levels, productivity measures (eg, number of patients seen) or process indicators (eg, percentage of diabetic patients receiving foot examinations), this instrument evaluates the level and nature of improvements made in the quality of chronic care delivery. ${ }^{21}$ The instrument has been identified as valid for assessing the quality of care delivery ${ }^{42}$ and valid and sensitive for tracing changes therein. ${ }^{43}$

The ACIC-S used in this study consists of 12 items covering the four key areas of the chronic care model: selfmanagement support $(\mathrm{n}=3)$, delivery system design $(n=3)$, decision support $(n=3)$ and clinical information systems $(n=3) \cdot{ }^{40}$ For each item, respondents selected the degree to which four descriptive levels of implementation, ranging from 'little or none' to 'fully implemented intervention', applied to their programmes. For example, with self-management support, the answering categories are: ' $\ldots$ is limited to the distribution of information (pamphlets, booklets) ', ... is available by referral to self-management classes or educators', '... is provided by trained clinical educators who are designated to do self-management support, are affiliated with each practice and see patients on referral', ' $\ldots$ is provided by clinical educators affiliated with each practice, trained in patient empowerment and problem-solving methodologies, and see most patients with chronic illness'. The result is a $0-11$ scale, with categories defined as $0-2$ (little or no support for chronic care), 3-5 (basic or intermediate support), 6-8 (advanced support) and 911 (optimal or comprehensive chronic care delivery). Subscale scores for the areas of the chronic care model were derived by calculating the average score for all items in each subsection when at least two of three items were available. The total quality of chronic care delivery score was calculated using the average scores for the four subsections (when at least three of four subsections were available). In the present study, Cronbach's $\alpha$ of the ACIC-S was 0.88 at baseline (T1) and 0.90 at T2 (1 year later), indicating reliability.

The team climate inventory (TCI) was used to measure professionals' perceptions of team climate while providing care to chronically ill adolescents. The questionnaire comprises four broad factors reflecting a team's shared perceptions of organisational policies, practices and procedures: shared vision and objectives (four items) (eg, To what extent do you think your team's objectives are clearly understood by other members of the team?), participative safety (four items) (eg, we have a 'we are in it together' attitude), task orientation (three items) (eg, Do members of the team build on each other's ideas in order to achieve the best possible outcome?) and support for innovation (three items) (eg, In this team we take the time needed to 
develop new ideas.). Participants were asked to rate their agreement with each TCI item on a five-point scale (1=strongly disagree, $2=$ disagree, $3=$ neither agree nor disagree, $4=$ agree and $5=$ strongly agree). Scores for each item in a scale are summed to determine the scale score. Higher scores indicated a better or more desirable team climate. ${ }^{44-46}$ In the present study, Cronbach's $\alpha$ of the TCI was 0.89 at baseline (T1) and 0.95 at T2 (1 year later), indicating reliability.

Respondents were also asked about personal/team characteristics, such as age, gender, occupation, number of years working in the organisation and number of working hours per week.

\section{Statistical analyses}

We used descriptive statistics to describe the study population. Two-tailed, paired t tests were used to investigate improvements in the quality of chronic care delivery and team climate over time. We employed a multilevel random-effects model to investigate the predictive role of (changes in) team climate on the quality of chronic care delivery at T2 while controlling for the quality of chronic care delivery at $\mathrm{T} 1$ and significant individual characteristics (significant at $\mathrm{p}<0.05$ in the univariate analyses) (SPSS V.20, mixed models option; SPSS, Inc, Chicago, Illinois, USA).

\section{RESULTS}

Women comprised $82 \%$ (of 144) of the respondents at T1. The mean age was $44.34 \pm 9.17$ (range 24-63) years. About half of the respondents (58\%) worked more than $29 \mathrm{~h} /$ week and $85 \%$ had been working in their current organisation for more than 3 years. Respondents consisted of nurses (39\%), specialists (31\%) (medical doctors eg, paediatrician, adolescent medicine specialist, rehabilitation specialist) and paramedical professionals $(7 \%)$, social workers $(11 \%)$, psychologists $(5 \%)$ and manager/quality officers $(7 \%)$.

The overall quality of the chronic care delivery (ACIC-S) score at T1 was 5.90, indicating basic or intermediate support for chronic care. Paired t test results showed significant improvement in the quality of chronic care delivery at T2. The mean ACIC-S score at T2 was 6.70, indicating advanced support for chronic care. The ACIC-S self-management support, decision support and clinical information systems subscales yielded similar results (table 1). No significant improvement was made in the delivery system design; the mean ACIC-S scores for this subscale were 7.37 at $\mathrm{T} 1$ and 7.64 at $\mathrm{T} 2$, both of which indicated advanced support for chronic care. In addition, no difference in team climate was found over time. Furthermore, changes in team climate and quality of chronic care delivery did not vary across teams. The mean score of team climate of 55.51 at $\mathrm{T} 1$ was substantially higher than Goh $e t a l \mathrm{~s}^{36}$ test group score of 50.40 in the primary care setting $(\mathrm{p} \leq 0.001)$.

We compared baseline characteristics of the respondents who completed both questionnaires to those who completed T1 only. No difference in team climate (TCI at T1) or overall quality of chronic care delivery (ACIC T1) was found.

We employed a multilevel random effects model to investigate the predictive role of (changes in) team climate on the quality of chronic care delivery at $\mathrm{T} 2$ while controlling for the quality of chronic care delivery at T1 and significant individual characteristics (significant at $\mathrm{p}<0.05$ in the univariate analyses). In the univariate analyses, age, gender, number of working hours per week and number of years working within the organisation were not significantly associated with quality of care delivery (results not shown). Regarding the respondent's occupation, only being a specialist was significantly associated with quality of care delivery $(\mathrm{r}=0.20 ; \mathrm{p}<0.05)$. Therefore, we only included respondent's occupation in the multilevel analyses with the largest group (nurse) as the reference category. After adjusting for the quality of chronic care delivery at $\mathrm{T} 1$ and respondent's occupation, multilevel analyses showed that team climate at $\mathrm{T} 1$ $(p<0.01)$ and changes in team climate $(p<0.001)$ predicted the quality of chronic care delivery at T2 (table 2).

\section{DISCUSSION}

This study aimed to (1) evaluate the effectiveness of implementing transition programmes in improving the

Table 1 Changes in the quality of chronic care delivery and team climate over time

\begin{tabular}{|c|c|c|c|c|c|c|c|c|}
\hline & \multicolumn{2}{|c|}{$\begin{array}{l}\text { Baseline } \\
\text { assessment } \\
(T 1,2010)\end{array}$} & \multicolumn{2}{|c|}{$\begin{array}{l}\text { Follow-up } \\
\text { assessment } \\
(\mathrm{T} 2,2011)\end{array}$} & \multicolumn{2}{|l|}{ T2-T1 } & \multirow[b]{2}{*}{ p Value* } & \multirow[b]{2}{*}{$\mathbf{n}$} \\
\hline & Mean & SD & Mean & SD & Mean & SD & & \\
\hline Overall quality of chronic care delivery (ACIC-S) & 5.90 & $(1.48)$ & 6.70 & $(1.20)$ & 0.79 & $(1.70)$ & $<0.001$ & 82 \\
\hline Self-management support (ACIC-S) & 5.35 & (1.79) & 6.91 & (2.42) & 1.57 & (2.36) & $<0.001$ & 86 \\
\hline Delivery system design (ACIC-S) & 7.37 & (1.93) & 7.64 & (2.51) & 0.27 & (2.25) & 0.271 & 85 \\
\hline Decision support (ACIC-S) & 5.59 & (2.07) & 6.03 & (2.20) & 0.44 & (2.07) & $<0.05$ & 82 \\
\hline Clinical information systems (ACIC-S) & 5.31 & (2.08) & 6.14 & (2.37) & 0.83 & (2.44) & $<0.01$ & 77 \\
\hline Team climate (TCI) & 55.51 & (6.09) & 55.91 & (9.01) & 0.40 & (8.94) & 0.689 & 82 \\
\hline
\end{tabular}

*Paired $t$ test, $\mathrm{T} 1 \mathrm{vs} \mathrm{T} 2$.

ACIC-S, assessment of chronic illness care short version; TCI, team climate inventory. 
Table 2 Predictors of chronic care delivery quality at T2 (random intercepts model, $n=78$ )

\begin{tabular}{|c|c|c|c|c|}
\hline & B & SD & B & SE \\
\hline Constant & $-1.63^{\star \star \star}$ & 1.57 & $6.67^{* * *}$ & 0.17 \\
\hline $\begin{array}{l}\text { Quality of chronic care } \\
\text { delivery at } \mathrm{T} 1 \text { (ACIC-S) }\end{array}$ & $0.68^{\star \star *}$ & 0.11 & $0.99^{* \star *}$ & 0.16 \\
\hline Specialists & $1.16^{\star *}$ & 0.38 & $0.43^{* *}$ & 0.14 \\
\hline Psychologists & 1.06 & 0.73 & 0.17 & 0.12 \\
\hline $\begin{array}{l}\text { Paramedical } \\
\text { professionals }\end{array}$ & 0.28 & 0.67 & 0.06 & 0.14 \\
\hline Social workers & 0.82 & 0.83 & 0.19 & 0.19 \\
\hline Manager/quality officer & 0.09 & 1.02 & 0.01 & 0.16 \\
\hline Team climate at $\mathrm{T} 1$ (TCl) & $0.07^{\star *}$ & 0.31 & $0.44^{\star \star}$ & 0.17 \\
\hline $\begin{array}{l}\text { Changes in team climate } \\
(\mathrm{TCl} ; \mathrm{T} 2-\mathrm{T} 1)\end{array}$ & $0.09^{* * *}$ & 0.22 & $0.79^{* * *}$ & 0.17 \\
\hline \multicolumn{5}{|c|}{$\begin{array}{l}{ }^{* * *} \mathrm{p} \leq 0.001 ;{ }^{* *} \mathrm{p} \leq 0.01 \text { (two-tailed). } \\
\text { Multilevel analyses included only data from respondents who filled } \\
\text { in questionnaires at T1 and T2. List wise deletion of missing } \\
\text { cases resulted in the inclusion of } 78 \text { cases in multilevel regression } \\
\text { analyses. The largest occupation group (nurse) is the reference } \\
\text { category. } \\
\text { ACIC-S, assessment of chronic illness care short version; TCl, } \\
\text { team climate inventory; T1=baseline (2010); T2=follow-up (2011). }\end{array}$} \\
\hline
\end{tabular}

quality of chronic care delivery, as described by the four key elements of the chronic care model; and (2) identify the predictive role of (changes in) team climate on the quality of chronic care delivery over time. Although expectations for various transitional interventions are largely agreed on, empirical evidence describing their effectiveness in improving the quality of chronic care delivery is lacking. ${ }^{2}$ Other studies of chronic care delivery (eg, in a primary care setting) have shown that multicomponent interventions are required to change delivery processes and outcomes. ${ }^{13-15} 20$ The chronic care model, a framework applied in multidisciplinary care settings to assess the quality of chronic care delivery, aims to transform this delivery from acute and reactive to proactive, integrated and personalised. To the best of our knowledge, this study was the first to investigate the effectiveness of transition programmes in improving the quality of chronic care delivery over time, as described by Wagner's chronic care model. ${ }^{19}$ Our results clearly showed significant improvements in the quality of chronic care delivery over time, from basic or intermediate support to advanced support for chronic care. One year after the start of the programme, responses in all four areas of the chronic care model as perceived by the professionals, as well as the overall quality of chronic care delivery, indicated advanced support for chronic care. Implementation of these transitional care programmes effectively reduced all bottlenecks identified by professionals at the start of the programme. Improvements were identified in coordination of care, access to resources for joint care services, having a joint mission, synchronising paediatric and adult treatment protocols, organising joint clinics and improving the provision of medical and psychosocial information about the transition period to patients. ${ }^{24}$ Reduction of these experienced bottlenecks may explain the improved quality of chronic care delivery over time.

We additionally investigated the predictive role of (changes in) team climate on the quality of chronic care delivery. We found that after adjusting for the quality of chronic care delivery at $\mathrm{T} 1$ and significant respondents' characteristics, team climate at the start of the programme and changes therein predicted the quality of chronic care delivery. These findings support our expectation that teams with positive and stimulating team climates, in which members share the same objectives, were better able to improve the quality of chronic care delivery. This stresses the importance of creating a positive and stimulating team climate among professionals from various disciplines. Healthcare professionals from different occupational backgrounds are known to have their own goals and priorities, ${ }^{47-49}$ which may compromise a stimulating team climate and hinder the team's ability to improve quality of care delivery.

While we found that team climate did not improve significantly over time in our study sample, at T1 team climate was already substantially higher compared to team climate in the primary care setting. ${ }^{36}$ Joining the transition programme may have led to improved team climate in the beginning of the programme but not over time.

We found that changes in team climate and the quality of chronic care delivery did not vary across teams targeting different patient populations (adolescents with type I diabetes, juvenile rheumatoid arthritis, cystic fibrosis, kidney conditions, urological conditions and neuromuscular disorders), suggesting that these mechanisms apply to all teams regardless of their patient population.

\section{CONCLUSIONS}

The implementation of transition programmes improves the quality of chronic care delivery as described by the chronic care model. Such implementation requires a supportive and stimulating team climate to enhance the quality of chronic care delivery to chronically ill adolescents in hospitals and rehabilitation centres.

Contributors APN drafted the design for data gathering. MS and AN were involved in the acquisition of participants and data; they also helped in the drafting of the manuscript and contributed to its refinement. JC drafted the manuscript and with AN performed the statistical analysis and interpretation of data. All authors have read and approved its final version.

Funding This study is funded with a grant from the Netherlands organisation for health research and development (ZonMw) grant number: 15700.2014.

\section{Competing interests None.}

Ethics approval This study was approved by the ethics committee of the Erasmus University Medical Centre, Rotterdam.

Provenance and peer review Not commissioned; externally peer reviewed. Data sharing statement No additional data are available.

Open Access This is an Open Access article distributed in accordance with the Creative Commons Attribution Non Commercial (CC BY-NC 3.0) license, which permits others to distribute, remix, adapt, build upon this work non- 
commercially, and license their derivative works on different terms, provided the original work is properly cited and the use is non-commercial. See: http:// creativecommons.org/licenses/by-nc/3.0/

\section{REFERENCES}

1. World Health Organization. Adolescent friendly services. Geneva: World Health Organization, 2002. http://whqlibdoc.who.int/hq/2003/ WHO FCH CAH 02.14.pdf

2. Forbes $\mathrm{A}$, While $\mathrm{A}$, Ullman $\mathrm{R}$, et al. A Multi-method review to identify the components of practice which may promote continuity in the transition from child to adult care for young people with chronic illness or disability. London: National Co-ordinating Centre for NHS Service Delivery and Organisation Research \& Development (NCCSDO), 2001.

3. Weissberg-Benchell J, Wolpert $\mathrm{H}$, Anderson JB. Transitioning from paediatric to adult care. Diabetes Care 2008;30:2441-6.

4. Rosen DS, Blum RW, Brotto M, et al. Society of adolescents medicine: transition to adult health care for adolescents and young adults with chronic conditions. J Adolesc Health 2003;33:309-11.

5. McDonagh JE, Kelly DA. The challenges and opportunities for transitional care research. Pediatr Transplant 2010;14:688-700.

6. While A, Forbes A, Ullman R, et al Good practices that address continuity during transition from child to adult care: synthesis of the evidence. Child Care Health Dev 2004;30:439-52.

7. Blum RW, Garell D, Hodgman $\mathrm{CH}$, et al. Transition from child centered to adult health-care systems for adolescents with chronic conditions. A position paper of the Society for Adolescent Medicine. J Adolesc Health 1993;14:570-6.

8. Blum RW. Transition to adult health care: setting the stage $J$ Adolesc Health 1995:17:3-5.

9. Scal P, Ireland M. Addressing transition to adult health care for adolescents with special health care needs. Pediatrics 2005;115:1607-12.

10. Sawyer SM, Blair S, Bowes G. Chronic illness in adolescents: transfer or transition to adult services? J Paediatr Child Health 1997;33:88-90.

11. McDonagh JE. Growing up and moving on: transition from pediatric to adult care. Pediatr Transplant 2005;9:364-72.

12. Reiss JG, Gibson RW, Walker LR. Health care transition: youth, family, and provider perspectives. Pediatrics 2005;115:112-20.

13. Von Korff M, Gruman J, Schaefer J, et al. Collaborative management of chronic illness. Ann Intern Med 1997;127:1097-102.

14. Wagner EH, Austin BT, Von Korff M. Improving outcomes in chronic illness. Manag Care Q 1996;4:12-25.

15. Wagner EH, Austin BT, Von Korff M. Organizing care for patients with chronic illness. Milbank Q 1996;74:511-44.

16. Maunder EZ. The challenge of transitional care for young people with life-limiting illness. Brit J Nurs 2004;13:594-6.

17. Watson AR. Non-compliance and transfer from paediatric to adult transplant unit. Pediatr Nephrol 2000;14:469-72.

18. Yeung E, Kay J, Roosevelt GE, et al. Lapse of care as a predictor for morbidity in adults with congenital heart disease. Int $J$ Cardiol 2008;125:62-5.

19. Wagner EH, Austin BT, Davis C, et al. Improving chronic illness care: translating evidence into action. Health Aff 2001;20:64-78

20. Coleman K, Austin BT, Brach C, et al. Evidence on the chronic care model in the new millennium. Health Aff 2009;28:75-85.

21. Bonomi AE, Wagner EH, Glasgow RE, et al. Assessment of chronic illness care $(\mathrm{ACIC})$ : a practical tool to measure quality improvement. BMC Health Serv Res 2002;37:791-820.

22. Britto MT, Vockell AL, Munafo JK, et al. Improving outcomes for underserved adolescents with asthma. Pediatrics 2014;133:e418-27.

23. van Staa A, van der Stege HA, Jedeloo S, et al. Readiness to transfer to adult care of adolescents with chronic conditions: exploration of associated factors. J Adolesc Health 2011;48:295-302.

24. Nieboer AP, Cramm JM, Sonneveld HM, et al. Reducing bottlenecks: professionals' and adolescents' experiences with transitional care delivery. BMC Health Serv Res 2014;14:47.

25. Pearson ML, Wu S, Schaefer J, et al. Assessing the implementation of the chronic care model in quality improvement collaboratives. Health Serv Res 2005;40:978-96.
26. Cramm JM, Nieboer AP. High-quality chronic care delivery improves experiences of chronically ill patients receiving care. Int $J$ Qual Healthcare 2013;25:689-95.

27. Tsai AC, Morton SC, Mangione CM, et al. A meta-analysis of interventions to improve care for chronic illnesses. Am J Manag Care 2005;11:478-88.

28. Adams SG, Smith PK, Allan PF, et al. Systematic review of the chronic care model in chronic obstructive pulmonary disease prevention and management. Arch Intern Med 2007;167:551-61.

29. Cramm JM, Adams SA, Walters BH, et al. The role of disease management programs in the health behavior of chronically ill patients. Patient Educ Couns 2014:87:411-15.

30. Cramm JM, Nieboer AP. Short and long term improvements in quality of chronic care delivery predict program sustainability. Soc Sci Med 2013;101:148-54.

31. Cramm JM, Nieboer AP. In the Netherlands, rich interaction among professionals conducting disease management led to better chronic care. Health Aff 2012;31:2493-500.

32. McDonagh JE, Minnaar G, Kelly K, et al. Unmet education and training needs in adolescent health of health professionals in a UK children's hospital. Acta Paediatr 2006;95:715-19.

33. Colquitt JA, Noe RA, Jackson CL. Justice in teams: antecedents and consequences of procedural justice climate in teams. Personnel Psychol 2002;55:83-109.

34. Bower $\mathrm{P}, \mathrm{Campbell} \mathrm{S}$, Bojke $\mathrm{C}$, et al. Team structure, team climate and the quality of care in primary care: an observational study. Qual Saf Health Care 2003;12:273-9.

35. Hann M, Bower P, Campbell S, et al. The association between culture, climate and quality of care in primary health care teams. Fam Pract 2007;24:323-9.

36. Goh TT, Eccles MP, Steen N. Factors predicting team climate, and its relationship with quality of care in general practice. $B M C$ Health Serv Res 2009;9:138.

37. Campbell SM, Hann M, Hacker J, et al. Identifying predictors of high quality care in English general practice: observational study. $B M J$ 2001;323:784-7.

38. Sonneveld HM, Strating MM, van Staa AL, et al. Gaps in transitional care: what are the perceptions of adolescents, parents and providers? Child Care Health Dev 2013;39:69-80.

39. Cramm JM, Strating MMH, Roebroeck ME, et al. The importance of general self-efficacy for the quality of life of adolescents with chronic conditions. Soc Indic Res 2013;113:551-61.

40. Cramm JM, Sonneveld $\mathrm{H}$, Strating MMH, et al. Higher satisfaction with transitional care improves social quality of life of chronically-ill adolescents. Appl Res Qual Life 2013;8:481-91.

41. Institute for Healthcare Improvement. The Breakthrough Series: IHI's Collaborative Model for Achieving Breakthrough Improvement. IHI Innovation Series white paper. Boston: Institute for Healthcare Improvement, 2003.

42. Cramm JM, Strating MMH, Tsiachristas A, et al. Development and validation of a short version of the assessment of chronic illness care $(\mathrm{ACIC})$ in Dutch disease management programs. Health Qual Life Out 2011;9:49.

43. Sunaert $\mathrm{P}$, Bastiaens $\mathrm{H}$, Nobbels $\mathrm{F}$, et al. Effectiveness of the introduction of a chronic care model-based program for type 2 diabetes in Belgium. BMC Health Serv Res 2010;10:207.

44. Anderson NR, West MA. Measuring climate for work group innovation: development and validation of the team climate inventory. J Organ Behav 1998;19:235-58.

45. Kivimäki M. Elovainio M. A short version of the team climate inventory: development and psychometric properties. J Occup Organ Psych 1999;72:241-6.

46. Strating $\mathrm{MMH}$, Nieboer AP. Psychometric test of the team climate inventory-short version investigated in Dutch quality improvement teams. BMC Health Serv Res 2009;9:126.

47. Rose Wiles JR. Teamwork in primary care: the views and experiences of nurses, midwives and health visitors. $J$ Adv Nurs 1994:20:324-30.

48. Lucas K, Bickler G. Altogether now? Professional differences in the priorities of primary care groups. J Public Health 2000;22:211-15.

49. Shaw A, de Lusignan $S$, Rowlands $G$. Do primary care professionals work as a team: A qualitative study. J Interprofes Care 2005;19:396-405 